Original

\title{
Leachables and cytotoxicity of root canal sealers
}

\author{
Indre Graunaite1), Greta Lodiene1), Odeta Arandarcikaite'), \\ Audrius Pukalskas'3), and Vita Machiulskiene1)
1)Department of Dental and Oral Pathology, Lithuanian University of Health Sciences, Kaunas, Lithuania
2)Institute of Neurosciences, Laboratory of Biochemistry, Lithuanian University of Health Sciences, Kaunas, Lithuania
${ }^{3)}$ Department of Food Science and Technology, Kaunas University of Technologies, Kaunas, Lithuania

(Received April 29, 2017; Accepted November 4, 2017)

\begin{abstract}
This in vitro study aimed to detect leaching components from an epoxy resin- and a methacrylatebased endodontic sealer and correlate them to cytotoxicity induced by material extracts for up to 36 weeks. We qualitatively determined the substances released by aged $\mathrm{AH}$ Plus and RealSeal SE specimens at seven intervals between 0 and 36 weeks. Quantification was performed by ultra-performance liquid chromatography/mass spectrometry (UPLC/MS). We determined the viability of murine macrophage J774 cells after $24 \mathrm{~h}$ exposure to material extracts, at each interval, using a fluorescence staining/microscopy method. The leachables detected were 1-adamantylamine and bisphenol A diglycidyl ether from AH Plus and $N$-( $p$-tolyl) diethanolamine and caprolactone-2(methacryloyloxy) ethyl ester from RealSeal SE. The largest UPLC/MS chromatogram peak areas of the leachables were detected within $72 \mathrm{~h}$. Induction of cytotoxicity after exposure to AH Plus and RealSeal SE extracts coincided with leachant detected within the first 72 and $24 \mathrm{~h}$, respectively. The clinical impact of the cytotoxicity due to resin-based endodontic sealers is unknown.
\end{abstract}

Keywords: cytotoxicity; chromatography; epoxy resins; mass spectrometry; root canal sealers.

Correspondence to Dr. Indre Graunaite, Lithuanian University of Health Sciences, Department of Dental and Oral Pathology, Eiveniu g. 2, Kaunas, LT-50161, Lithuania

E-mail: indre.graunaite@1smuni.lt

J-STAGE Advance Publication: August 12, 2018

Color figures can be viewed in the online issue at J-STAGE.

doi.org/10.2334/josnusd.17-0173

DN/JST.JSTAGE/josnusd/17-0173

\section{Introduction}

A number of root canal sealers are available, and their constituting materials determine their properties (degree of polymer conversion, induction of cytotoxicity, bond strength, antimicrobial effects, fracture resistance of root dentin, and osteogenic potential). Their incomplete polymerization and/or degradation may cause some components to leach from resin-based filling materials $(1,2)$ and root canal sealers $(3,4)$. Leaching from only a few root canal sealers has been detected by chromatography. However, no data about the leaching dynamics over time has been provided.

Epoxy- and methacrylate- based materials have various degrees of cytotoxicity before and after curing $(3,5)$. The leaching and toxicity profiles of root canal sealers are important because these are permanently implanted. Epoxy-based AH Plus (Dentsply International, York, PA, USA), methacrylate-based Epiphany (Pentron Clinical Technologies, LLCC, Wallingford, CT, USA), and EndoRez (Ultradent Products, Inc. South Jordan, UT, USA) sealers may be cytotoxic even up to one year after polymerization $(6,7)$. Our hypothesis is that resin-based sealers remain cytotoxic over time because of polymer degradation.

In the present study, leachables from two sealers were investigated by chromatographic methods at specific time-points after polymerization. In vitro cytotoxicity was tested by quantifying the exposure to the sealer material extracts collected.

\section{Materials and Methods}

Chemically cured AH Plus Jet (Dentsply DeTrey, 
Table 1 Composition of the root canal sealers tested (according to the manufacturers' datasheet).

\begin{tabular}{|c|c|}
\hline Root canal sealer & Chemical composition \\
\hline $\begin{array}{l}\text { AH Plus Jet (Dentsply DeTrey, Konstanz, Germany; } \\
\text { lot no: 1204001156) }\end{array}$ & $\begin{array}{l}\text { Epoxide paste: diepoxide, calcium tungstate, zirconium oxide, aerosil, } \\
\text { pigment. } \\
\text { Amine paste: } N, N \text { '-dibenzyl-5-oxa-nonandiamine-1,9, 1-adamantane } \\
\text { amine, tricylodecane-diamine, calcium tungstate, aerosil, zirconium } \\
\text { oxide, silicone oil. }\end{array}$ \\
\hline $\begin{array}{l}\text { RealSeal SE (SybronEndo, Orange, CA, USA; lot no: } \\
\text { 3616129) }\end{array}$ & $\begin{array}{l}\text { Mixture of EBPADMA, HEMA; Bis-GMA; acidic methacrylate resins, } \\
\text { barium boro silicate glasses, silica, hydroxyapatite, Ca-Al-F-silicate, } \\
\text { bismuth oxyclorite with amines, peroxide, photoinitiator, stabilizers, } \\
\text { pigment, aluminium oxide. }\end{array}$ \\
\hline
\end{tabular}

EBPADMA: ethoxylated bisphenol A dimethacrylate; HEMA: hydroxyethylmethacrylate; Bis-GMA: bisphenol A-glycidyl methacrylate.

Table 2 The structure, molecular weight $(M)$, mass/charge $(\mathrm{m} / \mathrm{z})$ values, and identification of the substances.

\begin{tabular}{lllll}
\hline ID & & & Identification \\
\hline 2 & & & \\
bisphenol A diglycidyl ether
\end{tabular}

I Identification letter in the chromatogram in Fig. 1.

Konstanz, Germany) and a dual-cured RealSeal SE (SybronEndo, Orange, CA, USA) root canal filling materials were chosen (Table 1).

Under aseptic conditions, uncured root canal sealer material was placed in cylindrical polytetrafluoroethylene (Guarniflon, Bergamo, Italy) molds of $2 \mathrm{~mm}$ height and $7 \mathrm{~mm}$ diameter. The dual-cured (i.e., lightand chemically cured) root canal sealer RealSeal SE was prepared according to the manufacturers' instructions. The light curing time was $40 \mathrm{~s}$ (Elipar FreeLight 2, 3M ESPE, St. Paul, MN, USA, with a built-in irradiance indicator; irradiance $1,000 \mathrm{~mW} / \mathrm{cm}^{2}$; wavelength range $430-480 \mathrm{~nm}$; maximum irradiance at $455 \mathrm{~nm}$ ) (Nordic Institute of Dental Materials. Tested depth of cure and curing lamps. Table 2 [updated April 1st 2014; cited February 11th 2017]. Available from: http://niom.no/ tested-depth-of-cure-and-curing-lamps-table-2/). The chemically cured AH Plus was similarly molded, but not light-cured. Eight discs (four for each material) were prepared and stored inside a dark container at $37^{\circ} \mathrm{C}$ and $95 \%$ relative humidity for $24 \mathrm{~h}$ to allow complete setting.

Ultra-performance liquid chromatography/mass spectrometry (UPLC/MS) and cytotoxicity assessment of the extracts were performed after aging the specimens for 24 $\mathrm{h}$ at the following time-points: $0-24 \mathrm{~h} ; 24-72 \mathrm{~h} ; 72 \mathrm{~h}$ to 1 week; 1-4 weeks; 4-12 weeks; $12-24$ weeks; and 24-36 weeks (Fig. 1). For the latter four aging periods, samples were taken following 1 week extraction time, which was the last week of the aging periods. The procedure for collecting leachables for the first $0-24 \mathrm{~h}$ was as follows: each test specimen of each material was placed in 968 $\mu \mathrm{L}$ of sterile phosphate-buffered saline (PBS, $\mathrm{pH}$ 7.4; Sigma-Aldrich, St. Paul, MO, USA) in eppendorf tubes and incubated for $24 \mathrm{~h}$ at $37^{\circ} \mathrm{C}$ under agitation. The ratio of the specimen surface area to the PBS extraction medium volume was $1.25 \mathrm{~cm}^{2} / \mathrm{mL}$ (8). PBS was replaced 


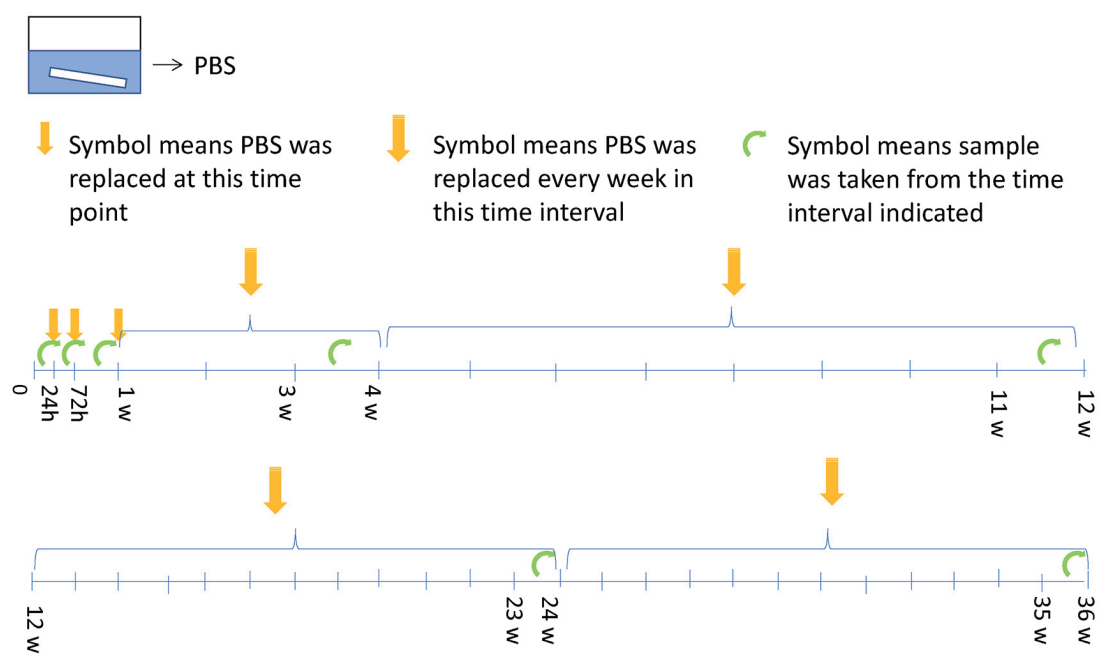

Fig. 1 Design of aging and extract procedure. Flowchart for one specimen. Aging time of material after $24 \mathrm{~h}$ setting time.

after $24 \mathrm{~h}, 72 \mathrm{~h}, 1$ week, and every week thereafter under strict aseptic conditions to avoid fungal or bacterial contamination (6). For aging periods longer than 1 week, the samples represented the last week of the aging period (i.e., the time between weeks 3-4, 11-12, 23-24, and 35-36) (Fig. 1). The aging experiments were performed using four specimens: one specimen was used for chromatography analysis and three were used for cytotoxicity assessment. Extracts obtained from the different aging periods, to be investigated for chromatographic analysis and cytotoxicity assessment, were filtered using the extraction medium from each eppendorf tube containing the specimen (sterile filter $0.22 \mu \mathrm{m}$, Millex GP, Millipore, Carrigtwohill, Co. Cork, Ireland). The samples used for cytotoxicity assessment were diluted with cell culture medium (see section 1.4) for a final concentration of 1:2 ( $\mathrm{vol} / \mathrm{vol}$ ) (extract:medium). These concentrations were selected to assess the cytotoxicity within a detectable toxicity range.

Chromatography was performed using an Acquity UPLC system (Waters, Milford, MA, USA). It consisted of a binary solvent delivery pump, an autosampler with a $10 \mu \mathrm{L}$ sample loop, a photodiode array detector, and a column manager. A Bruker maXis UHR-TOF mass spectrometer (Bruker Daltonics, Bremen, Germany) was used for an accurate mass measurement. Zorbax C18 columns $(5 \mu \mathrm{m}, 150 \times 4.6 \mathrm{~mm}$, inner diameter; Agilent Technologies, Santa Clara, CA, USA) were used for separation of compounds at $25^{\circ} \mathrm{C}$. The separation was carried out under isocratic conditions, using methanol/ water (Sigma-Aldrich) (80:20; v/v) buffered with 40 $\mathrm{mM}$ ammonium formate (Sigma-Aldrich). The flow rate of the mobile phase was $0.8 \mathrm{~mL} / \mathrm{min}$ and split in two parts: $0.6 \mathrm{~mL} / \mathrm{min}$ to waste and $0.2 \mathrm{~mL} / \mathrm{min}$ to the mass spectrometer equipped with an electrospray ionization source. Instrument control and data acquisition were achieved using the Compass 1.3 software (HyStar 3.2 SR2) (Bruker Daltonics, Bremen, Germany). MS experiments were performed in the positive ionization mode, maintaining the capillary voltage at $4,500 \mathrm{~V}$ with the end plate offset at $-500 \mathrm{~V}$.

Nitrogen was used as the drying and nebulizing gas, at a flow rate of $10.0 \mathrm{~L} / \mathrm{min}$ and a pressure of 2.0 bar. Mass spectra were recorded at a range from 50 to 1,200 mass $/$ charge $(\mathrm{m} / \mathrm{z})$, at a rate of $5.0 \mathrm{~Hz}$. Peak identification was carried out by comparing the retention times with those of the corresponding peaks in chromatograms of standards (1-adamantylamine, Sigma-Aldrich), and the extraction medium (PBS) or by analyzing the measured accurate $\mathrm{m} / \mathrm{z}$ values (mass accuracy $1 \mathrm{ppm}$ ), calculated molecular formulas, and obtained specific fragments. The limit of the detection method for 1-adamantylamine was estimated to be $27 \mathrm{ng} / \mathrm{mL}$. Comparison of the eluted substances was performed by calculating the peak areas of the corresponding masses of each substance. The peak areas were integrated using the Bruker Quant Analysis software (Bruker Daltonics). The amount of each substance was assumed to be linearly proportional to the area under its peak. Concentrations of the substances other than 1-adamantylamine were calculated in relative values.

The murine macrophage $\mathrm{J} 774$ cell line (passages 1-25) was maintained in Dulbecco's Modified Eagle's Medium (DMEM; Sigma-Aldrich), supplemented with $10 \%$ fetal calf serum (Sigma-Aldrich) and $50 \mu \mathrm{g} / \mathrm{mL}$ of gentamicin (Sigma-Aldrich) at $37^{\circ} \mathrm{C}$ in humidified atmosphere 
A

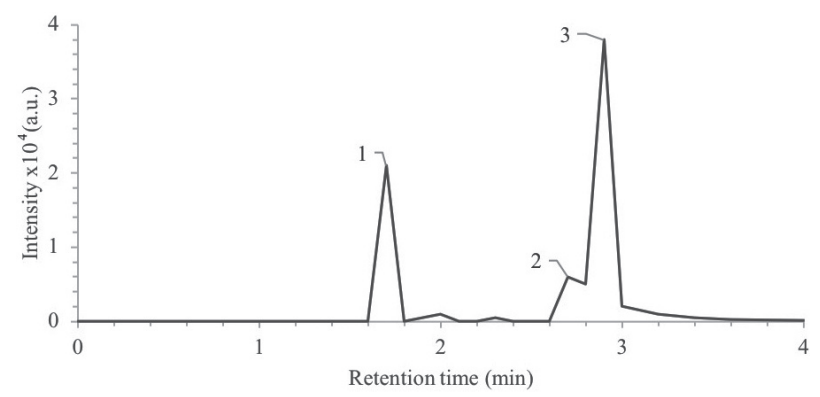

B

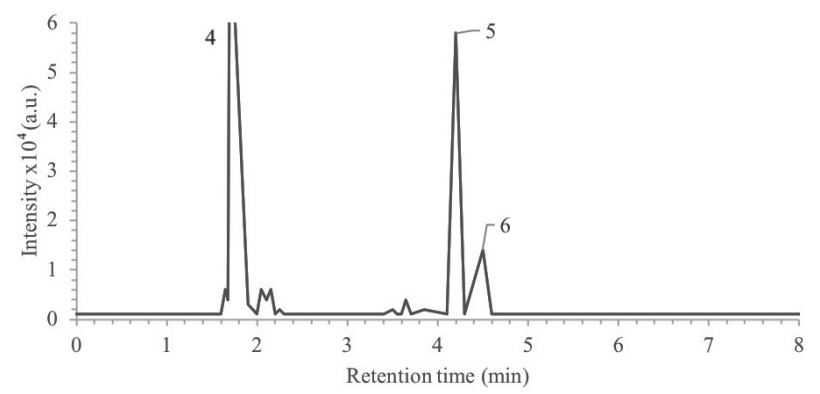

Fig. 2 Ultra-Performance liquid chromatography/mass spectrometry (UPLC/MS) chromatograms of extracts from root canal sealer materials after aging in PBS for $24 \mathrm{~h}$. (A) AH Plus (B) RealSeal SE. Peaks 1 and 4 originated from PBS solution used as extraction medium and were not further analyzed; peak 2- bisphenol A diglycidyl ether; peak 3- 1-adamantylamine; peak 5- $N$-( $p$ tolyl) diethanolamine; peak 6- caprolactone 2-(methacryloyloxy) ethyl ester (see Table 2). Data from one experiment.

containing $5 \% \mathrm{CO}_{2}$. Percentage cell viability and total cell number were measured using the trypan blue exclusion method and Bürke counting chamber. The cells (initial culture density of $3 \times 10^{5} \mathrm{~mL}^{-1}$ ), grown in a monolayer, were suspended in cell culture medium and seeded in 24-well tissue culture plates (Falcon, Becton Dickinson Lab., Franklin Lakes, NJ, USA) and incubated for $24 \mathrm{~h}$ prior to exposure. Twenty thousand cells were seeded in each well. Three independent experiments, with tissue culture plates prepared separately, were performed. The same concentrations were used for each material and aging time. Cells were exposed to the extraction medium for $24 \mathrm{~h}$.

Cell viability was assessed using a modification of the method described by Lodiene et al. (3). Cells were stained with propidium iodide (PI)/Hoechst 33342 and visualized using an Olympus IX71 fluorescent microscope (Olympus Europe, Hamburg, Germany, excitation wavelength $350 \mathrm{~nm}$; optical zoom 100×). A minimum of 400 cells was counted from each sample. Evenly red-stained and blue-stained cells with condensed, fragmented nuclei were classified as dead cells (necrotic or apoptotic). Evenly blue-stained cells were categorized
A

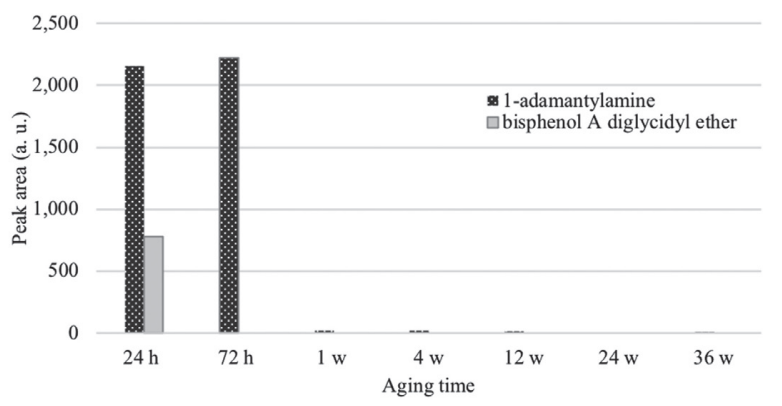

B

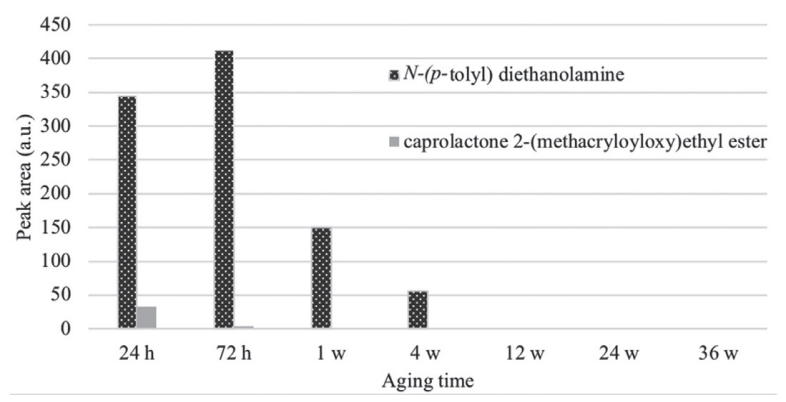

Fig. 3 Semi-quantitative determination of eluted substances at the different material aging periods, after setting for $24 \mathrm{~h}$, expressed as histogram of peak areas (arbitrary units). The area is proportional to the amount of the substance. Substances eluted from samples of (A) AH Plus (B) RealSeal SE. Data from one experiment.

as viable. Three experiments with two parallels of each exposed and control samples were performed with extracts from each material and each aging period. Cells in DMEM culture medium for $24 \mathrm{~h}$ were used as control.

ANOVA analysis with Fisher's least significant difference test was performed using the SPSS 22 statistical software package (SPSS, Chicago, IL, USA). A $P$ value of $<0.05$ was considered statistically significant. The significance level was verified by the nonparametric Kruskal-Wallis and Mann-Whitney tests.

\section{Results}

The chromatograms revealed two major peaks each in the AH Plus (Fig. 2A) and RealSeal SE (Fig. 2B) samples aged for $24 \mathrm{~h}$. The structure, molecular weight, $\mathrm{m} / \mathrm{z}$ values, and identification of the eluted substances are shown in Table 2.

Peaks of 1-adamantylamine and bisphenol A diglycydyl ether (BADGE) were observed in the chromatograms of the extracts from AH Plus (Fig. 2A) obtained from the $0-72 \mathrm{~h}$ and the $0-24 \mathrm{~h}$ intervals, respectively (Fig. 3A). A lower percent of the 1-adamantylamine was present in the $72 \mathrm{~h}$ to 1 week interval, and traces were detected up to 


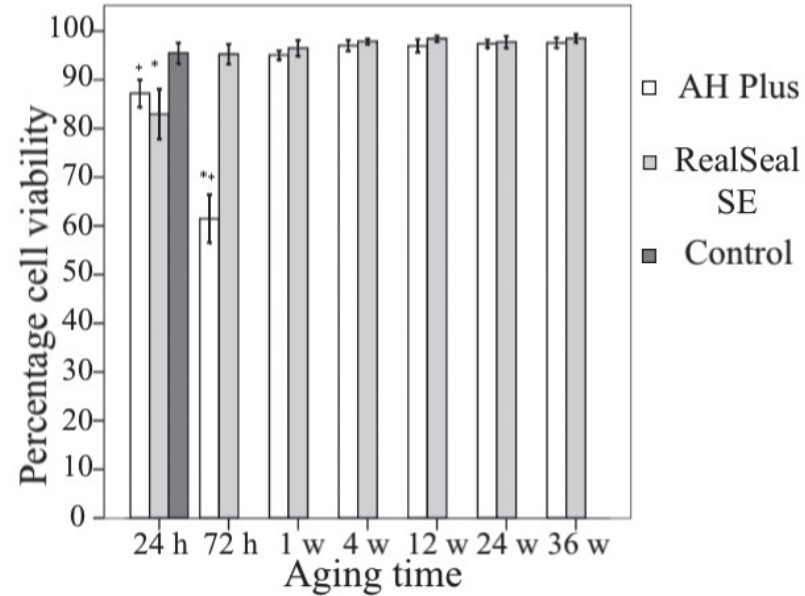

Fig. 4 Cell viability of J774 cell culture after $24 \mathrm{~h}$ incubation with AH Plus and RealSeal SE extracts obtained from different aging times and extraction periods compared with the DMEM control. * denotes significant difference from control; + denotes significant difference between AH Plus and RealSeal SE $(P<$ 0.001). Dilution was 1:2 (extract:medium) for all exposed samples.

36 weeks. Quantification of the eluted substances could not be performed because the substances were not available as individual reference materials. Therefore, in the present study, we used a relative comparison by calculating and comparing the area of substance chromatogram peaks. The peak area of $N$-( $p$-tolyl) diethanolamine in the UPLC/MS chromatogram of RealSeal SE 0-24 h sample (Fig. 2B) was reduced to $\sim 50 \%$ in the $72 \mathrm{~h}$ to 1 week sample. This compound was also detected in the 3-4 weeks aging period extract (Fig. 3B). After mass spectra analysis, small peak areas $(<10 \%$ of that of the amine) of caprolactone 2-(methacryloyloxy) ethyl ester were observed in the RealSeal SE chromatograms from samples with aging periods 0-24 h (Fig. 2B) and 24-72 h, after which no traces were observed (Fig. 3B).

Cell death was induced after exposure to material extracts from $\mathrm{AH}$ Plus and RealSeal SE specimens aged up to 72 and $24 \mathrm{~h}$, respectively $(P<0.05$, relative to control) (Fig. 4). The cytotoxicity of AH Plus and RealSeal SE was significantly different in the samples obtained from the 24-72 $\mathrm{h}$ aging period $(P<0.001)$.

\section{Discussion}

For many years, chromatography has been the first choice for the analysis of components in polymer-based dental materials and eluted substances (9). LC/MS is a widely used chromatographic method $(10,11)$. The compounds 1-adamantylamine and BADGE were previously detected in uncured AH Plus samples (3). No data about leachables from RealSeal SE was found in the literature. A limitation of our study was the single experiment performed for the chromatographic investigation. The identified substances $N$-( $p$-tolyl) diethanolamine and caprolactone 2-(methacryloyloxy) ethyl ester are an amine used for the activation of the polymerization and a side product, respectively. Our finding showing that only two peaks could be seen in each chromatogram agreed with previous studies that evaluated the degree of conversion of the same root canal sealers. Specifically, both AH Plus and RealSeal SE exhibited a high degree of conversion in these studies (12-14). A thorough curing procedure of the samples under laboratory conditions may have contributed to an increased degree of conversion, compared with the clinical situation, and may partly account for the limited elution. Elution of the substances is also dependent on the type of extraction media (15). Extraction solvents, such as water, ethanol, saliva, or PBS, can be used in the chromatographic analysis of dental resin materials $(4,16,17)$. Higher concentrations of the extracts and additional chemicals could be expected if ethanol was used as the extraction medium $(9,16)$. However, PBS was chosen in this study to simulate an in vivo contact with the periapical tissues and to make the samples suitable for the further cytotoxicity testing.

Previous studies have used other cell cultures to investigate AH Plus and RealSeal SE root canal sealer cytotoxicity in vitro, the most commonly ones being fibroblasts and osteoblasts (6). Macrophage cell lines such as $\mathbf{J 7 7 4}$ or Raw 264.7 are well-established model systems in cell biology and immunology $(18,19)$. Previous studies investigating the mechanism of action of methacrylate-based root canal sealers cytotoxicity indicated that eluted resin components caused an increase in intracellular reactive oxygen species and decreased intracellular glutathione concentration (20).

Our results showed a decrease in cytotoxicity of extracts from AH Plus and RealSeal SE after $72 \mathrm{~h}$ aging. This data is in line with results from several other studies $(21,22)$. However, long-term in vitro cytotoxicity studies, using MC3T3 osteoblasts and THP1 monocytes, have shown that tested materials remained cytotoxic for up to one year (7). Differences in cytotoxicity level and duration between studies may be explained by differences in study models, i.e., whether cells were exposed to eluates or were in direct contact with the material. A longer contact time between specimens and cells or the use of different cell cultures also affected the results. Root canal sealers are intended to stay within root canal space, but they can sometimes extrude through the apical foramina. This implies that in vivo periapical tissues can have a contact 
with both the sealer and its eluates. Our finding (low cytotoxicity in the 0-24 h samples) is in agreement with the chromatographic analysis: no cytotoxic monomers such as bisphenol A-glycidyl dimethacrylate (Bis-GMA) (23) or 2-hydroxyethylmethacrylate (HEMA) (24) eluted from the tested samples (Table 1). However, a drop in the cell viability induced by the 24-72 h AH Plus eluate combined with no changes in the chromatogram of the eluate sample for the corresponding aging period indicated the presence of eluting materials beyond the detection limits. Bis-GMA, HEMA, and ethoxylated bisphenol methacrylate (EBPADMA) were found to be cytotoxic $(23,24)$ and were found to leach from other root canal filling materials (25). These monomers may have eluted from RealSeal SE sealer if different extraction media had been used.

Leaching of substances from the materials may influence the healing process through inflammation and thereby cause a less than optimal clinical outcome. Root canal sealers have been shown to affect histopathological repair of periodontal tissues in dogs (26). Increased inflammation due to root canal obturation materials may result in more acute postoperative pain. Data from clinical trials indicate that overfilling is associated with postoperative pain (27) that can be caused by the core, the sealer material, or by the combination of both. Furthermore, the choice of different sealers can potentially affect the intensity and duration of the pain. As shown recently in an in vitro study, $\mathrm{AH}$ Plus evokes greater trigeminal neuronal activity compared with control, while RealSeal SE reduces it (28).

Only a few clinical studies have investigated the outcome of root canal treatment with different obturation materials. A retrospective clinical study of endodontic treatment's long-term outcome showed that teeth obturated using a combination of Resilon/RealSeal SE had significantly higher chances of failure than those obturated with gutta-percha/AH Plus (29).

A large number of studies on leaching of dental polymer-based restorative materials have been published, but to date, data on root canal polymers are scarce. Current in vitro findings have indicated that sealers do leach after polymerization and cause a cytotoxic reaction, which decreases with time. The degree of reversibility of this primary adverse effect is largely unknown. Furthermore, the mutagenicity of several polymer-based restorative materials has been shown in vitro (Scientific Committee on Emerging and Newly Identified Health Risks [SCENIHR], Scientific opinion on the Safety of Dental Amalgam and Alternative Dental Restoration Materials for Patients and Users, 6 May 2008. https:// ec.europa.eu/health/scientific_committees/emerging/ docs/scenihr_o_046.pdf).

However, aside from reported allergies to some substances, there is limited information on possible toxicity and adverse health effects of polymer-based restorative materials (https://ec.europa.eu/health/scientific_committees/emerging/docs/scenihr_o_046.pdf).

Current clinical studies investigating the effect of root canal sealer on periapical healing are not informative due to poor study design or small sample size. Therefore, whether leaching and in vitro cytotoxicity have any clinical significance is unclear. Cytotoxicity studies are part of the biocompatibility tests suggested in ISO 7405 for such materials and an important starting point for comparisons.

\section{Acknowledgments}

The financial support of the Research Foundation of Lithuanian University of Health Sciences is gratefully acknowledged. The authors thank Dr. Ellen Bruzell (Nordic Institute of Dental Materials) for help in editing the manuscript and Dr. Hilde Kopperud (Nordic Institute of Dental Materials) and Dr. Egle Arbaciauskiene (Kaunas University of Technology) for assistance with the chromatographic analysis.

\section{References}

1. Darmani H, Al-Hiyasat AS, Milhem MM (2007) Cytotoxicity of dental composites and their leached components. Quintessence Int 38, 789-795.

2. Imazato S, Horikawa D, Nishida M, Ebisu S (2009) Effects of monomers eluted from dental resin restoratives on osteoblastlike cells. J Biomed Mater Res B Appl Biomater 88, 378-386.

3. Lodienė G, Kopperud HM, Ørstavik D, Bruzell EM (2013) Detection of leachables and cytotoxicity after exposure to methacrylate- and epoxy-based root canal sealers in vitro. Eur J Oral Sci 121, 488-496.

4. Furche S, Hickel R, Reichl FX, van Landuyt K, Shehata M, Durner J (2013) Quantification of elutable substances from methacrylate based sealers and their cytotoxicity effect on with human gingival fibroblasts. Dent Mater 29, 618-625.

5. Karapınar-Kazandağ M, Bayrak OF, Yalvac ME, Ersev H, Tanalp J, Sahin F et al. (2011) Cytotoxicity of 5 endodontic sealers on L929 cell line and human dental pulp cells. Int Endod J 44, 626-634.

6. Brackett MG, Messer RL, Lockwood PE, Bryan TE, Lewis JB, Bouillaguet S et al. (2010) Cytotoxic response of three cell lines exposed in vitro to dental endodontic sealers. J Biomed Mater Res B Appl Biomater 95, 380-386.

7. Brackett MG, Lewis JB, Kious AR, Messer RL, Lockwood PE, Brackett WW et al. (2012) Cytotoxicity of endodontic sealers after one year of aging in vitro. J Biomed Mater Res B Appl Biomater 100, 1729-1735.

8. International Organization of Standardization (2012) 
Biological evaluation of medical devices. ISO 10993:2012, Geneve.

9. Van Landuyt KL, Nawrot T, Geebelen B, De Munck J, Snauwaert J, Yoshihara K et al. (2011) How much do resinbased dental materials release? A meta-analytical approach. Dent Mater 27, 723-747.

10. Durner J, Obermaier J, Draenert M, Ilie N (2012) Correlation of the degree of conversion with the amount of elutable substances in nano-hybrid dental composites. Dent Mater 28, 1146-1153.

11. Ilie N, Obermaier J, Durner J (2014) Effect of modulated irradiation time on the degree of conversion and the amount of elutable substances from nano-hybrid resin-based composites. Clin Oral Investig 18, 97-106.

12. Peutzfeldt A (1997) Resin composites in dentistry: the monomer systems. Eur J Oral Sci 105, 97-116.

13. Wu WC, Shrestha D, Wei X, Ling JQ, Zhang WH, Chen J (2010) Degree of conversion of a methacrylate-based endodontic sealer: a micro-Raman spectroscopic study. J Endod 36, 329-333.

14. Baldi JV, Bernardes RA, Duarte MA, Ordinola-Zapata R, Cavenago BC, Moraes JC et al. (2012) Variability of physicochemical properties of an epoxy resin sealer taken from different parts of the same tube. Int Endod J 45, 915-920.

15. Ferracane JL (1994) Elution of leachable components from composites. J Oral Rehabil 21, 441-452.

16. Kopperud HM, Schmidt M, Kleven IS (2010) Elution of substances from a silorane-based dental composite. Eur J Oral Sci 118, 100-102.

17. Durner J, Spahl W, Zaspel J, Schweikl H, Hickel R, Reichl FX (2010) Eluted substances from unpolymerized and polymerized dental restorative materials and their Nernst partition coefficient. Dent Mater 26, 91-99.

18. Kant AM, De P, Peng X, Yi T, Rawlings DJ, Kim JS et al. (2002) SHP-1 regulates Fcgamma receptor-mediated phagocytosis and the activation of RAC. Blood 100, 1852-1859.

19. Touret N, Paroutis P, Terebiznik M, Harrison RE, Trombetta S, Pypaert M et al. (2005) Quantitative and dynamic assess- ment of the contribution of the ER to phagosome formation. Cell 123, 157-170.

20. Engelmann J, Volk J, Leyhausen G, Geurtsen W (2005) ROS formation and glutathione levels in human oral fibroblasts exposed to TEGDMA and camphorquinone. J Biomed Mater Res B Appl Biomater 75, 272-276.

21. Silva EJ, Santos CC, Zaia AA (2013) Long-term cytotoxic effects of contemporary root canal sealers. J Appl Oral Sci 21, 43-47.

22. Zhou HM, Du TF, Shen Y, Wang ZJ, Zheng YF, Haapasalo $M$ (2015) In vitro cytotoxicity of calcium silicate-containing endodontic sealers. J Endod 41, 56-61.

23. Hanks CT, Strawn SE, Wataha JC, Craig RG (1991) Cytotoxic effects of resin components on cultured mammalian fibroblasts. J Dent Res 70, 1450-1455.

24. Yoshii E (1997) Cytotoxic effects of acrylates and methacrylates: relationships of monomer structures and cytotoxicity. $\mathrm{J}$ Biomed Mater Res 37, 517-524.

25. Omurlu H, Arisu HD, Dalkilic EE, Tamer U, Torul H (2016) Investigation of eluted monomers from resin-based root canal sealer by high-performance liquid chromatography analysis. Eur J Dent 10, 92-96.

26. Tanomaru Filho M, Leonardo MR, Silva LA, Utrilla LS (1998) Effect of different root canal sealers on periapical repair of teeth with chronic periradicular periodontitis. Int Endod J 31, 85-89.

27. Gesi A, Hakeberg M, Warfvinge J, Bergenholtz G (2006) Incidence of periapical lesions and clinical symptoms after pulpectomy--a clinical and radiographic evaluation of 1versus 2-session treatment. Oral Surg Oral Med Oral Pathol Oral Radiol Endod 101, 379-388.

28. Ruparel NB, Ruparel SB, Chen PB, Ishikawa B, Diogenes A (2014) Direct effect of endodontic sealers on trigeminal neuronal activity. J Endod 40, 683-687.

29. Barborka BJ, Woodmansey KF, Glickman GN, Schneiderman E, He J (2017) Long-term clinical outcome of teeth obturated with resilon. J Endod 43, 556-560. 